\title{
One-Step Predictive H2 FIR Tracking under Persistent Disturbances and Data Errors
}

\author{
OSCAR IBARRA-MANZANO, JOSE ANDRADE-LUCIO, YURIY S. SHMALIY \\ Department of Electronics Engineering, University of Guanajuato Salamanca, 36885, MEXICO \\ YUAN XU \\ School of Electrical Engineering, University of Jinan, Jinan 250022, CHINA
}

\begin{abstract}
Information loss often occurs in industrial processes under unspecified impacts and data errors. Therefore robust predictors are required to assure the performance. We design a one-step H2 optimal finite impulse response (H2-OFIR) predictor under persistent disturbances, measurement errors, and initial errors by minimizing the squared weighted Frobenius norms for each error. The H2-OFIR predictive tracker is tested by simulations assuming Gauss-Markov disturbances and data errors. It is shown that the H2-OFIR predictor has a better robustness than the Kalman and unbiased FIR predictor. An experimental verification is provided based on the moving robot tracking problem.
\end{abstract}

Keywords: Industrial errors, object tracking, H2 FIR predictor, Kalman predictor, unbiased FIR predictor.

Received: February 7, 2021. Revised: July 30, 2021. Accepted: August 16, 2021. Published: September 5, 2021.

\section{Introduction}

Predictive tracking refers to the process of predicting the future object position [1]. Robust predictive control is required in vision robot tracking [2], wireless sensor networks (WSNs) [3], power grids [4], and smart sensing [5]. It is also provided under missing data caused by latency and propagation losses [6], [7] and in different kinds of industrial motors to enhance robustness against parameter perturbations [8].

To organize prediction, one can employ an estimate $\hat{x}_{k}$ at discrete time index $k$ and project it ahead using the system matrix $F$ as $\hat{x}_{k+1}=F \hat{x}_{k}$ that yields an accurate estimate under the unknown future noise [9]-[12], When some data are temporary unavailable, then future observation $y_{k+1}$ can be predicted as $y_{k+1}=H F \hat{x}_{k}$, where $H$ is the observation matrices. A drawback is that recursive forms available for white noise (diagonal error matrices) produce more errors than batch estimators operating with full error matrices [13]-[20] An advantage of FIR structures is the bounded input bounded output stability [16]. A disadvantage is the computational complexity $\mathcal{O}\left(N^{2}\right)$ inherent to batch estimators. However unlike in Kalman's days, the computational complexity is not already an issue for modern computers [21].

The recursive $\mathrm{H}_{2}$ approach was extensively investigated in [22]-[27] and generalized in [28]-[30]. Since the $H_{2}$ problem is convex, it has a closed form solution resulting in the $\mathrm{KF}$ under Gaussian noise [29]. It worth noting that, as argued in [20], the RH $H_{2}$ FIR filter can perform similarly to the RH $H_{\infty}$ filter. Even so, the standard $H_{2}$ FIR approach [16] has two critical drawbacks: 1) the squared Frobenius norm is minimized unweighted and 2) data errors and initial errors are ignored. That requires further investigations.

In this paper, we derive a one-step $\mathrm{H}_{2}$ optimal FIR $\left(\mathrm{H}_{2}\right.$ OFIR) predictor for robust predictive tracking under persistent disturbances, data errors, and initial errors. By simulations and experimentally, we show that the $\mathrm{H}_{2}$-OFIR predictor operating with full error matrices is a more robust estimator than the Kalman predictor (KP) and unbiased FIR (UFIR) predictor [32], [33].

\section{State Space Model}

Consider a process represented in discrete-time state-space with the following linear equations

$$
\begin{aligned}
x_{k+1} & =F x_{k}+E u_{k}+B w_{k}, \\
y_{k} & =H x_{k}+D w_{k}+v_{k},
\end{aligned}
$$

where $x_{k} \in \mathbb{R}^{K}, u_{k} \in \mathbb{R}^{L}, y_{k} \in \mathbb{R}^{P}, F \in \mathbb{R}^{K \times K}$, $H \in \mathbb{R}^{P \times K}, E \in \mathbb{R}^{K \times L}, B \in \mathbb{R}^{K \times M}$, and $D \in \mathbb{R}^{P \times M}$. Vector $w_{k} \in \mathbb{R}^{M}$ represents a disturbance and $v_{k} \in \mathbb{R}^{P}$ is a measurement error; both zero mean, bounded, and with uncertain distributions and covariances.

Expand model (1) and (2) on a horizon $[m, k]$ of $N$ points, where $m=k-N+1$, as

$$
\begin{aligned}
x_{k+1} & =F^{N} x_{m}+\bar{S}_{N} U_{m, k}+\bar{D}_{N} W_{m, k}, \\
Y_{m, k} & =H_{N} x_{m}+L_{N} U_{m, k}+T_{N} W_{m, k}+V_{m, k},
\end{aligned}
$$

where $U_{m, k}=\left[u_{m}^{T} u_{m+1}^{T} \ldots u_{k}^{T}\right]^{T}, \quad W_{m, k}=$

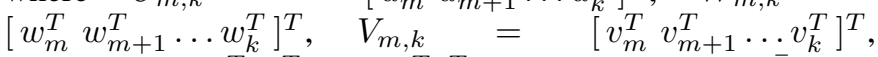
$Y_{m, k}=\left[y_{m}^{T} y_{m+1}^{T} \ldots y_{k}^{T}\right]^{T}$, and $H_{N}=\bar{H}_{N} F_{N}$, $L_{N}=\bar{H}_{N} S_{N}, T_{N}=G_{N}+\bar{T}_{N}, G_{N}=\bar{H}_{N} D_{N}$,

$$
\begin{aligned}
F_{N}= & {\left[\begin{array}{cccccccc}
I & F^{T} & \ldots & F^{N-2^{T}} & F^{N-1^{T}}
\end{array}\right]^{T}, } \\
S_{N}= & {\left[\begin{array}{cccccc}
0 & 0 & \ldots & 0 & 0 & 0 \\
E & 0 & \ldots & 0 & 0 & 0 \\
F E & E & \ldots & 0 & 0 & 0 \\
\vdots & \vdots & \ddots & \vdots & \vdots & \vdots \\
F^{N-3} E & F^{N-4} E & \ldots & E & 0 & 0 \\
F^{N-2} E & F^{N-3} E & \ldots & F E & E & 0
\end{array}\right], }
\end{aligned}
$$


$\bar{T}_{N}=\operatorname{diag}(\underbrace{D D \ldots D}_{N})$, and $\bar{H}_{N}=\operatorname{diag}(\underbrace{H H \ldots H}_{N})$. Matrix $D_{N}$ is equal to matrix $S_{N}$ if we substitute $E$ with $B, \bar{S}_{N}$ is the last row vector in $S_{N}$ and so is $\bar{D}_{N}$ in $D_{N}$.

\section{Optimal H2 FIR Predictor}

To derive a batch $\mathrm{H}_{2}$-OFIR predictor, we refer to (4) and define the FIR predicted estimate as

$$
\begin{aligned}
\hat{x}_{k+1}= & \mathcal{H}_{N} Y_{m, k}+\mathcal{H}_{N}^{\mathrm{f}} U_{m, k}, \\
= & \mathcal{H}_{N} H_{N} x_{m}+\left(\mathcal{H}_{N} L_{N}+\mathcal{H}_{N}^{\mathrm{f}}\right) U_{m, k} \\
& +\mathcal{H}_{N} T_{N} W_{m, k}+\mathcal{H}_{N} V_{m, k},
\end{aligned}
$$

where $\mathcal{H}_{N}$ is the fundamental gain and $\mathcal{H}_{N}^{\mathrm{f}}$ is the forced gain. The unbiasedness condition $\mathcal{E}\left\{x_{k+1}\right\}=\mathcal{E}\left\{\hat{x}_{k+1}\right\}$ applied to (3) and (5) yields the unbiasedness constraints

$$
\begin{aligned}
I & =\mathcal{H}_{N} \mathbf{C}_{N}, \\
\mathcal{H}_{N}^{\mathrm{f}} & =\bar{S}_{N}-\mathcal{H}_{N} L_{N},
\end{aligned}
$$

where $\mathbf{C}_{N}=H_{N} F^{-(N-1)}$. We next define the prediction error as $\varepsilon_{k+1}=x_{k+1}-\hat{x}_{k+1}$ and introduce the bias error residual matrix $\mathcal{B}_{N}$, system error residual matrix $\mathcal{W}_{N}$, and observation error residual matrix $\mathcal{V}_{N}$ as

$$
\begin{aligned}
\mathcal{B}_{N} & =F^{N}-\mathcal{H}_{N} H_{N}, \\
\mathcal{W}_{N} & =\bar{D}_{N}-\mathcal{H}_{N} T_{N}, \\
\mathcal{V}_{N} & =\mathcal{H}_{N}
\end{aligned}
$$

to represent the prediction error $\varepsilon_{k+1}$ as

$$
\begin{aligned}
\varepsilon_{k+1}= & \mathcal{B}_{N} x_{m}+\left(\bar{S}_{N}-\mathcal{H}_{N} L_{N}-\mathcal{H}_{N}^{\mathrm{f}}\right) U_{m, k} \\
& +\mathcal{W}_{N} W_{m, k}-\mathcal{V}_{N} V_{m, k},
\end{aligned}
$$

which, subjected to (7), can finally be generalized with

$$
\varepsilon_{k+1}=\varepsilon_{x(k+1)}+\varepsilon_{w(k+1)}+\varepsilon_{v(k+1)},
$$

where the sub-errors are given by $\varepsilon_{x(k+1)}=x_{m}-\hat{x}_{m}=$ $\mathcal{B}_{N} x_{m}, \varepsilon_{w(k+1)}=\mathcal{W}_{N} W_{m, k}$, and $\varepsilon_{v(k+1)}=-\mathcal{V}_{N} V_{m, k}$.

\subsection{Disturbance-to-Error Transfer Function}

The $\mathrm{H}_{2}$ performance is guaranteed by minimizing the trace of the squared Frobenius norm $\|\mathcal{T}(z)\|_{F}^{2}$ of the transfer function $\mathcal{T}(z)$ averaged over all frequencies [28]. That means minimizing effects of the $x_{m}$-to- $\varepsilon_{k+1}$ transfer function $\mathcal{T}_{x}(z)$, $w_{k}$-to- $\varepsilon_{k+1}$ transfer function $\mathcal{T}_{w}(z)$, and $v_{k}$-to- $\varepsilon_{k+1}$ transfer function $\mathcal{T}_{v}(z)$ at the estimator output.

Since the initial state error $\varepsilon_{x(k+1)}$ goes to $\varepsilon_{k+1}$ directly, then it follows that

$$
\mathcal{T}_{x}(z)=I .
$$

The $w_{k}$-to- $\varepsilon_{k+1}$ transfer function $\mathcal{T}_{w}(z)$ can be found by representing $W_{m, k}$ as [16], [24]

$$
W_{m, k}=A_{w} W_{m-1, k-1}+B_{w} w_{k},
$$

where the strictly sparse block matrices are constructed as

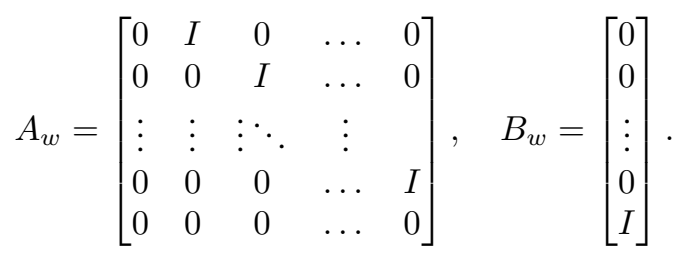

Then the transform applied to (14) yields $w(z)=(I z-$ $\left.A_{w}\right)^{-1} B_{w} w(z)$ and the transform applied to error $\varepsilon_{w(k+1)}=$ $\left(\bar{D}_{N}-\mathcal{H}_{N}^{\mathrm{h}} T_{N}\right) w_{m, k}$ results in the transfer function

$$
\mathcal{T}_{w}(z)=\mathcal{W}_{N}\left(I z-A_{w}\right)^{-1} B_{w} .
$$

Similarly, the transfer function of $V_{m, k}$ can be found to be

$$
\mathcal{T}_{v}(z)=\mathcal{V}_{N}\left(I z-A_{w}\right)^{-1} B_{w} .
$$

To minimize the prediction error, effects of the transfer functions (13), (16), and (17) must be minimized in the predictor error (12) as will be shown next.

\subsection{H2-OFIR Predictor}

We now introduce a product $\mathcal{T}(z) \varpi_{k}$ of $\mathcal{T}(z)$ and some proper vector $\varpi_{k}$. The weighted squared Frobenius norm can be defined by averaging over both variables $z$ and $k$ as [34]

$$
\begin{aligned}
\|\mathcal{T}(z)\|_{F}^{2}= & \mathcal{E}_{z}\left\{\mathcal{E}_{k}\left\{\operatorname{tr}\left[\mathcal{T}(z) \varpi_{k} \varpi_{k}^{*} \mathcal{T}^{*}(z)\right]\right\}\right\} \\
= & \frac{1}{2 \pi} \int_{0}^{2 \pi} \operatorname{tr}\left[\mathcal{T}\left(e^{j \omega T}\right) \mathcal{E}\left\{\varpi_{k} \varpi_{k}^{*}\right\}\right. \\
& \left.\times \mathcal{T}^{*}\left(e^{j \omega T}\right)\right] \mathrm{d} \omega T,
\end{aligned}
$$

where $\mathcal{T}^{*}(z)$ is a conjugate transpose of $\mathcal{T}(z)$ and $\mathcal{E}\left\{\varpi_{k} \varpi_{k}^{*}\right\}$ for each error can be assigned as

$$
\begin{aligned}
\mathcal{B}_{N} \chi_{m} \mathcal{B}_{N}^{T} & =\mathcal{B}_{N} \chi_{m} \mathcal{B}_{N}^{T}, \\
\mathcal{Q}_{N} & =\mathcal{E}\left\{W_{k, m} W_{k, m}^{T}\right\}, \\
\mathcal{R}_{N} & =\mathcal{E}\left\{V_{k, m} V_{k, m}^{T}\right\},
\end{aligned}
$$

where $\chi_{m}=\mathcal{E}\left\{x_{m} x_{m}^{T}\right\}$. If we now combine (19) and (18) and provide the averaging over $\omega T$, then the weighted norm $\mathcal{T}_{x}$ can be significantly simplified and written as

$$
\left\|\mathcal{T}_{x}(z)\right\|_{F}^{2}=\mathcal{B}_{N} \chi_{m} \mathcal{B}_{N}^{T} .
$$

Next, the weighted norm of $\mathcal{T}_{w}$ can be written using (20) as

$$
\begin{aligned}
\left\|\mathcal{T}_{w}(z)\right\|_{F}^{2}= & \frac{1}{2 \pi} \int_{0}^{2 \pi} \operatorname{tr}\left[\mathcal{T}_{w}\left(e^{j \omega T}\right) \mathcal{Q}_{N}\right. \\
& \left.\times \mathcal{T}_{w}^{*}\left(e^{j \omega T}\right)\right] \mathrm{d} \omega T .
\end{aligned}
$$

To arrive at a closed form of (23), we first suppose that $\mathcal{Q}_{N}=$ $I$ and transform (23) to $\left\|\mathcal{T}_{w}(z)\right\|_{F}^{2}=\operatorname{tr}\left(\mathcal{W}_{N} \mathcal{P} \mathcal{W}_{N}^{T}\right)$, where matrix $\mathcal{P}$ is a solution to the Lyapunov equation

$$
\mathcal{P}=A_{w} \mathcal{P} A_{w}^{T}+M,
$$

in which $M$ is allowed to be any positive definite matrix. We thus assign $M=B_{w} \mathcal{Q}_{N} B_{w}^{T}$ and represent a solution to (24) as $\mathcal{P}=\sum_{i=0}^{\infty} A_{w}^{i} B_{w} \mathcal{Q}_{N} B_{w}^{T} A_{w}^{T^{i}}$ [35] that, for $A_{w}$ and $B_{w}$ given 
by (15), readily becomes $\mathcal{P}=\mathcal{Q}_{N}$. Consequently, $\left\|\mathcal{T}_{w}(z)\right\|_{F}^{2}$ transforms to

$$
\left\|\mathcal{T}_{w}(z)\right\|_{F}^{2}=\operatorname{tr}\left(\mathcal{W}_{N} \mathcal{Q}_{N} \mathcal{W}_{N}^{T}\right) .
$$

The weighted norm of $\mathcal{T}_{v}$ can be found similarly as

$$
\left\|\mathcal{T}_{v}(z)\right\|_{F}^{2}=\operatorname{tr}\left(\mathcal{V}_{N} \mathcal{R}_{N} \mathcal{V}_{N}^{T}\right)
$$

and the trace of the error matrix $P=E\left\{\varepsilon_{k} \varepsilon_{k}^{T}\right\}$ for mutually uncorrelated errors becomes

$$
\begin{aligned}
\operatorname{tr} P= & E\left\{\left(\varepsilon_{x(k+1)}+\varepsilon_{w(k+1)}+\varepsilon_{v(k+1)}\right)^{T}(\ldots)\right\} \\
= & \operatorname{tr}\left(\mathcal{B}_{N} \chi_{m} \mathcal{B}_{N}^{T}+\mathcal{W}_{N} \mathcal{Q}_{N} \mathcal{W}_{N}^{T}\right. \\
& \left.+\mathcal{V}_{N} \mathcal{R}_{N} \mathcal{V}_{N}^{T}\right) .
\end{aligned}
$$

Because the $H_{2}$ problem is convex [35], find the gain $\mathcal{H}_{N}$ using (27) by considering the cost function

$$
J=\underset{\mathcal{H}_{N}}{\arg \min } \operatorname{tr} P\left(\mathcal{H}_{N}\right) .
$$

By setting the derivative $\frac{\partial}{\partial \mathcal{H}_{N}} J$ to zero, come up with

$$
\begin{aligned}
0= & -2\left(F^{N} \chi_{m} H_{N}^{T}+\bar{D}_{N} \mathcal{Q}_{N} G_{N}^{T}\right) \\
& +2 \mathcal{H}_{N}\left(H_{N} \chi_{m} H_{N}^{T}+G_{N} \mathcal{Q}_{N} G_{N}^{T}+\mathcal{R}_{N}\right)
\end{aligned}
$$

that results in the $\mathrm{H}_{2}$-OFIR predictor gain

$$
\begin{aligned}
\mathcal{H}_{N}= & \left(F^{N} \chi_{m} H_{N}^{T}+\bar{D}_{N} \mathcal{Q}_{N} G_{N}^{T}\right) \\
& \times\left(H_{N} \chi_{m} H_{N}^{T}+G_{N} \mathcal{Q}_{N} G_{N}^{T}+\mathcal{R}_{N}\right)^{-1}
\end{aligned}
$$

where the error matrix $\mathcal{Q}_{N}$ is computed by (21), $\mathcal{R}_{N}$ by (22), and other block matrices are defined after (4).

It can now be shown that the $\mathrm{H}_{2}$-OFIR predictor generalizes the OFIR predictor in a special case of Gaussian processes in the following batch estimate

$$
\hat{x}_{k+1}=\mathcal{H}_{N} Y_{m, k}+\left(\bar{S}_{N}-\mathcal{H}_{N} L_{N}\right) U_{m, k},
$$

where $\mathcal{H}_{N}$ is specified by (30). The error matrix $P_{k+1}=$ $\mathcal{E}\left\{\varepsilon_{k+1} \varepsilon_{k+1}^{T}\right\}$ of the $H_{2}$-OFIR predictor can be shown to be

$$
P_{k+1}=\mathcal{B}_{N} \chi_{m} \mathcal{B}_{N}^{T}+\mathcal{W}_{N} \mathcal{Q}_{N} \mathcal{W}_{N}^{T}+\mathcal{V}_{N} \mathcal{R}_{N} \mathcal{V}_{N}^{T}
$$

where the error residual matrices are defined above.

\section{Simulations}

To investigate the $\mathrm{H}_{2}$-OFIR predictor properties, we will think that the robot dynamics and measurements are corrupted by colored noise. Accordingly, we represent the robot and its observation along a coordinate $\mathrm{x}$ with a two-state space tracking model

$$
\begin{aligned}
x_{k+1} & =F x_{k}+B w_{k}, \\
w_{k} & =\phi w_{k-1}+\zeta_{k}, \\
v_{k} & =\psi v_{k-1}+\xi_{k}, \\
y_{k} & =H x_{k}+v_{k},
\end{aligned}
$$

where matrices are assigned as

$$
F=\left[\begin{array}{ll}
1 & \tau \\
0 & 1
\end{array}\right], \quad B=\left[\begin{array}{c}
\tau / 2 \\
1
\end{array}\right], \quad H=\left[\begin{array}{ll}
1 & 0
\end{array}\right]
$$

TABLE I

RMSES UNDER THE GAUSS-MARKOV DISTURBANCES

\begin{tabular}{lccc}
\hline$N_{\text {opt }}, \phi, \psi$ & \multicolumn{3}{c}{ RMSE, m } \\
\cline { 2 - 4 } & $H_{2}$-OFIR & UFIR & KF \\
\hline $26,0,0$ & 4.1818 & 4.1802 & $\mathbf{4 . 0 4 8 2}$ \\
\hline $10,0.95,0$ & $\mathbf{6 . 4 1 6 9}$ & 6.5657 & 9.1782 \\
\hline $55,0,0.95$ & 37.433 & 34.836 & $\mathbf{3 3 . 5 7 4}$ \\
\hline $3,0.95,0.95$ & 37.167 & $\mathbf{3 6 . 1 8 7}$ & 48.870 \\
\hline
\end{tabular}

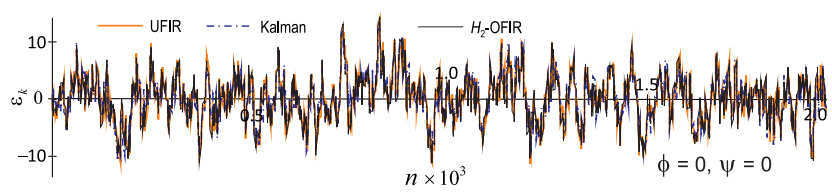

(a)

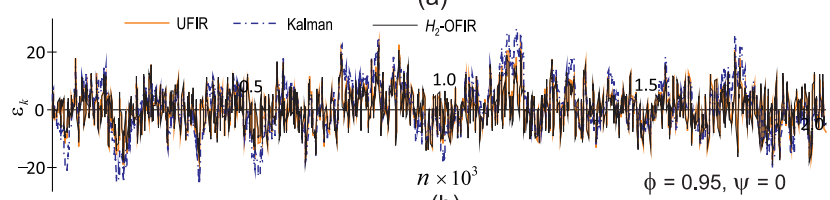

(b)

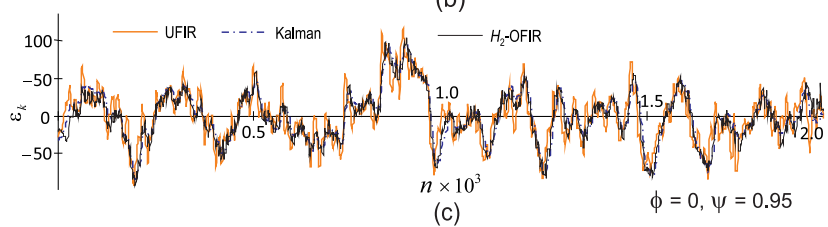

(c)

Fig. 1. Estimation errors produced by the $\mathrm{H}_{2}$-OFIR, UFIR, and Kalman predictors in Gaussian environments: (a) $\phi=\psi=0$, (b) $\phi=0.95$ and $\psi=0$, and (c) $\phi=0$ and $\psi=0.95$.

with $\tau=0.5 \mathrm{~s}$. Vectors $w_{k}$ and $v_{k}$ are chosen to be stable Gauss-Markov processes (34) and (35) with the scalar factors $0 \leqslant \phi<1$ and $0 \leqslant \psi<1$. The white Gaussian driving sources are set as $\zeta_{k} \sim \mathcal{N}\left(0, \sigma_{\varsigma}^{2}\right)$ and $\xi_{k} \sim \mathcal{N}\left(0, \sigma_{\xi}^{2}\right)$, The goal is to learn effects produced by the colored process noise (CPN) and colored measurement noise $(\mathrm{CMN})$ on the $\mathrm{H}_{2}$ OFIR predictor performance.

The case of Gaussian errors is favorable for KP. To compare the root mean square errors (RMSEs) under the Gauss-Markov disturbances, we set $\sigma_{\zeta}=0.3 \mathrm{~m} / \mathrm{s}$ and $\sigma_{\xi}=10 \mathrm{~m}$, simulate the processes for extreme values of $\phi$ and $\psi$, measure the optimal horizon $N_{\text {opt }}$ for the UFIR predictor [33], compute full block error matrices $\mathcal{Q}_{N}$ and $\mathcal{R}_{N}$, set $Q=\left(\mathcal{Q}_{N}\right)_{1,1}$ and $R=\left(\mathcal{R}_{N}\right)_{1,1}$ to the $\mathrm{KP}$, run the algorithms, and list the RMSEs in Table I, where the minimum errors are bolded. Examples of the estimation errors are sketched in Fig. 1.

As expected, the KP performs better when $\phi=\psi=0$, although the difference with other predictors appears to be insignificant (Table I). A situation changes dramatically under the sever disturbance with $\phi=0.95$. Here all FIR structures perform very consistently, while the KP produces of about $30 \%$ larger errors. An explanation to this results can be found in the nonzero components of the error matrix $\mathcal{Q}_{N}$ when $\phi=$ 0.95 and the fact that the KP discards the cross components, which become nonzero when noise is not white. An example 


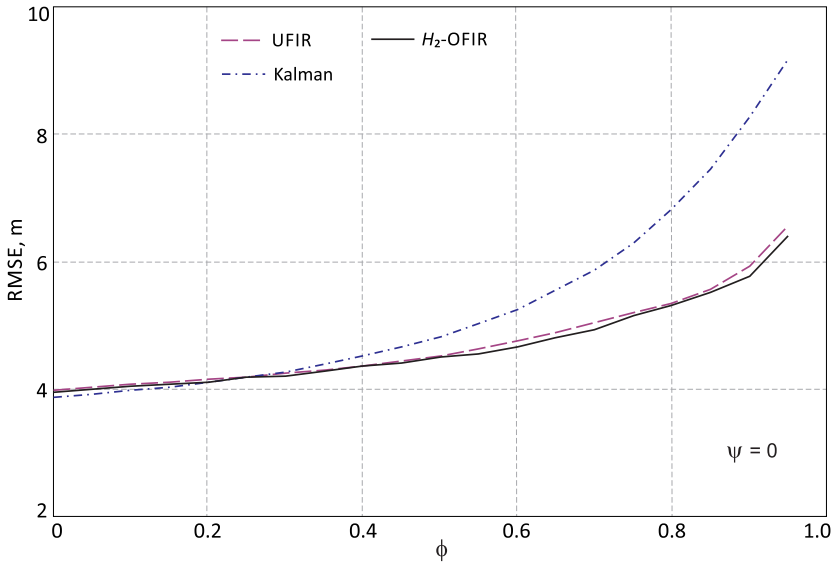

Fig. 2. RMSEs produced by the $\mathrm{H}_{2}$-OFIR, UFIR, and Kalman estimators with $\psi=0$ as functions of factor $\phi$ under the Gauss-Markov disturbances.

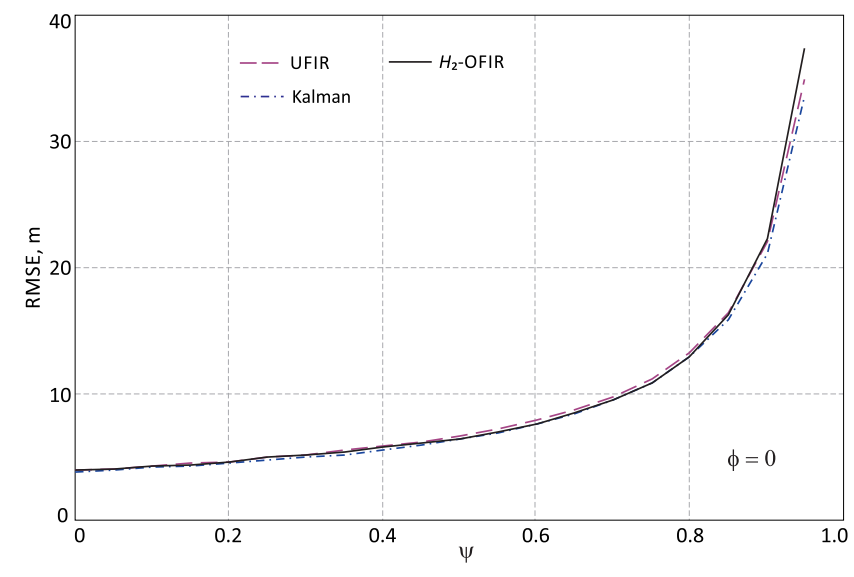

Fig. 3. RMSEs produced by the $\mathrm{H}_{2}$-OFIR, UFIR, and Kalman estimators with $\phi=0$ as functions of factor $\psi$ of the Gauss-Markov disturbance.

of matrix $\mathcal{Q}_{N}$ measured for $N=5$ and $\phi=0.95$,

$$
\mathcal{Q}_{N}=\left[\begin{array}{lllll}
1.0882 & 1.0425 & 0.9990 & 0.9575 & 0.9169 \\
1.0425 & 1.0895 & 1.0436 & 1.0001 & 0.9584 \\
0.9990 & 1.0436 & 1.0904 & 1.0446 & 1.0009 \\
0.9575 & 1.0001 & 1.0446 & 1.0914 & 1.0453 \\
0.9169 & 0.9584 & 1.0009 & 1.0453 & 1.0920
\end{array}\right]
$$

reveals even more: all of the components are of the same order of magnitude and thus neglecting the cross components will cause errors. The effect is negligible when $\phi$ is small and it becomes brightly pronounced when $\phi$ approaches unity (Fig. 2). However, this inference does not hold for the GaussMarkov disturbances (Fig. 3), in which case all the predictors produce consistent error, although the $\mathrm{H}_{2}$-OFIR predictor still performs better. We explain it by the observation that the disturbed state is required to be tracked, while the GaussMarkov noise needs to be filtered out that is better provided by other algorithms [36], [37]. A conclusion one can arrive at is that the $\mathrm{H}_{2}$-OFIR and UFIR predictors are more robust trackers than the KP.

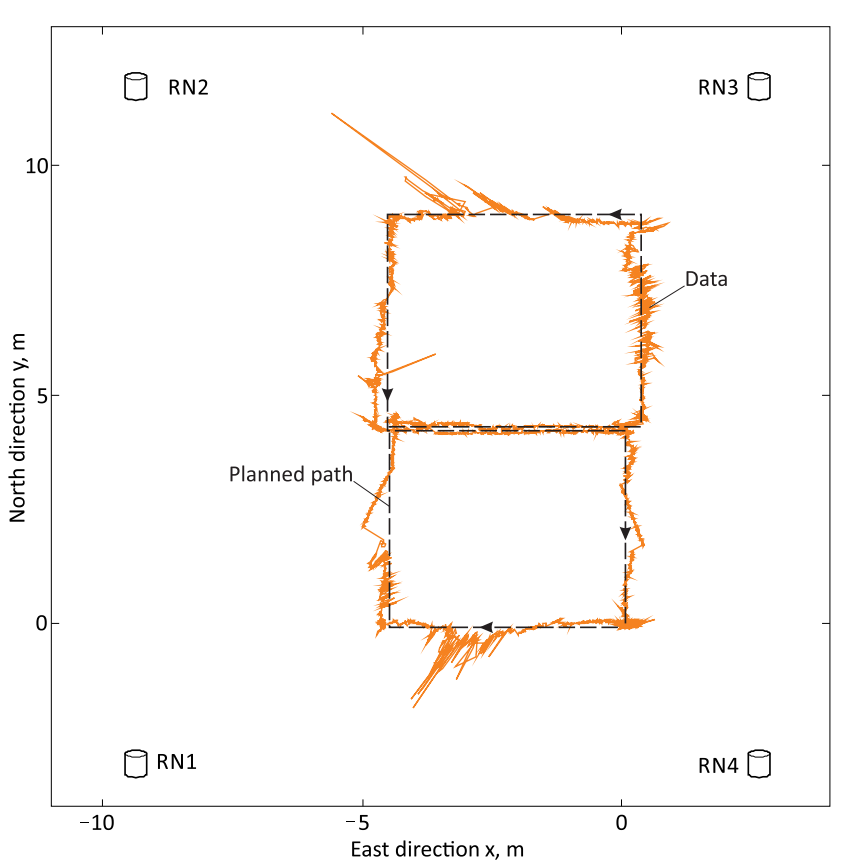

Fig. 4. UWB-based measurement data of a robot travelling along a planned path (dashed) in the indoor environment with four reference nodes deployed as RN1...RN4.

\section{Robot Predictive Tracking}

To verify the $H_{2}$-OFIR predictor performance, we have organized an experimental set and conducted measurements of a moving robot platform in the indoor environment of the Machine Building of the University of Jinan, Jinan, China. Data were obtained with $\tau=0.05 \mathrm{~s}$ using the ultra wide band (UWB) technology as described in [38].

Four reference nodes (RNs) were deployed with known coordinates as shown in Fig. 4: RN1 $(-9.4,-3) \mathrm{m}$, RN2 $(-9.4,11.78) \mathrm{m}$, RN3 $(2.63,11.78) \mathrm{m}$, and RN4 $(2.63,-3) \mathrm{m}$. The planned path trajectory along the east coordinate $\mathrm{x}$ is shown in Fig. 5 together with the UWB data. It is seen that some data jump from one point to another, while some others are reminiscent of the Gaussian and uniformly-distributed noise. There are also several outliers associated with the Cauchy noise. We therefore model data errors as the GaussMarkov disturbance, compute the difference with the planned path, and measure the full error matrix $\mathcal{R}_{N}$. The robot velocity in this experiment is about $0.4 \mathrm{~m} / \mathrm{s}$ and we accept $\sigma_{w}=0.1 \mathrm{~m} / \mathrm{s}$, set $Q=0.01 \mathrm{~m} / \mathrm{s}$, specify the diagonal matrix $\mathcal{Q}_{N}$ with all components equal to $Q$, and measure $N_{\text {opt }}=30$. That allows evaluating the estimator robustness against the $\mathrm{CMN}$ and CPN.

The RMSEs of predictive tracking are sketched in Fig. 6 for the initial path of 1100 points. It is seen that the KP and UFIR predictor perform consistently with the RMSEs of $0.1268 \mathrm{~m}$ (UFIR) and $0.1270 \mathrm{~m}(\mathrm{KP})$ and demonstrate the well-known transient effects [33] around $k=500$. In turn, the $\mathrm{H}_{2}$-OFIR estimator operating with full matrix $\mathcal{R}_{N}$ produces smaller 


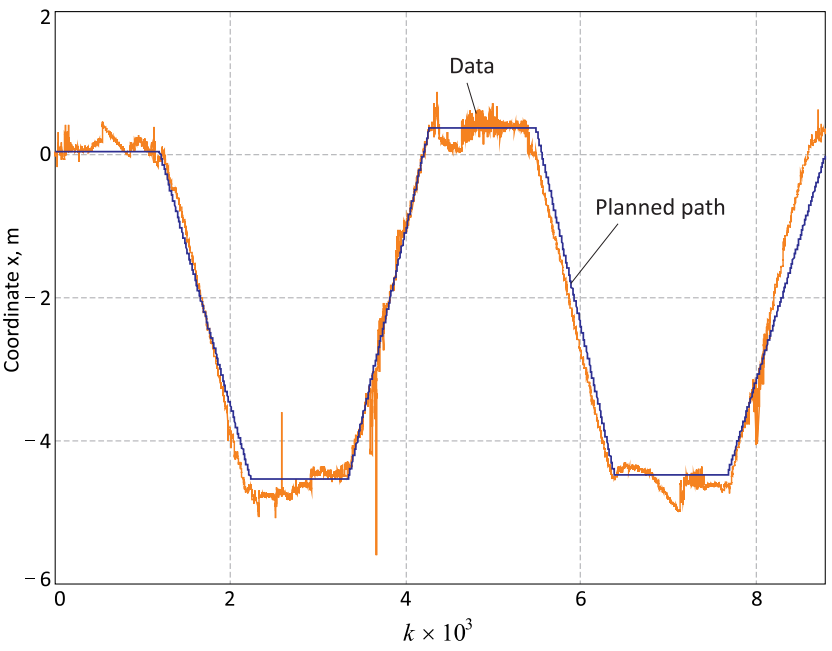

Fig. 5. The planned path along the coordinate $\mathrm{x}$ of a robot moving in an indoor environment and measurement data provided using the UWB technology with four RNs.

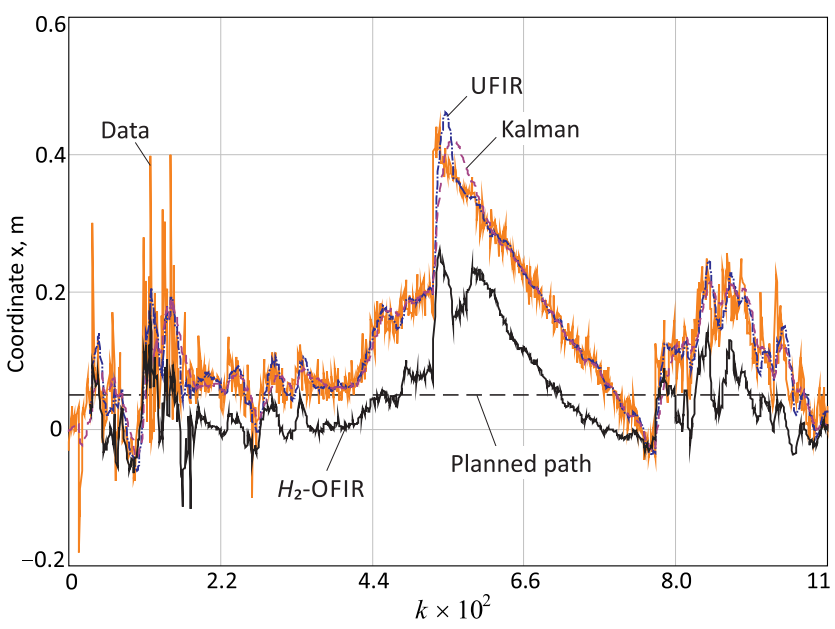

Fig. 6. Measurement data and predictive tracking estimates produced by the $\mathrm{H}_{2}$-OFIR, UFIR, and Kalman predictors at the beginning of the planned path (dashed) trajectory shown in Fig. 5.

RMSEs of $0.0665 \mathrm{~m}$ and ranges closer to the planned path. This gives an evidence of a better accuracy and robustness of the batch $\mathrm{H}_{2}$-OFIR predictor.

\section{Conclusions}

The $\mathrm{H}_{2}$-OFIR predictor derived in this paper under bounded industrial persistent disturbances, data errors, and initial errors has demonstrated a better performance and robustness than the KP and UFIR predictor. Namely, the $\mathrm{H}_{2}$-OFIR predictor operating with full error matrices has appeared to be more robust than the Kalman and UFIR predictors. The effect has been achieved by improving the derivation procedure and minimizing the squared weighted Frobenius norms at the predictor output. The performance of the $\mathrm{H}_{2}$-OFIR predictor was investigated by simulating the Gauss-Markov environment associated with industrial operation conditions. Thereby, it has been confirmed that the derived predictor is more robust than the KP and UFIR predictor. An experimental verification was provided for a moving robot travelling along a planned path in an indoor environment using the UWB technology.

We are now working on a more general $\mathrm{H}_{2}$-OFIR predictor for uncertain industrial processes observed under the disturbances, data errors, and initial errors and plan to report the results in near future.

\section{References}

[1] P. P. Kanjilal, Adaptive Prediction and Predictive Control, London, UK: Peter Peregrinus, 1995.

[2] A. Liu, W. Zhang, and L. Yu, "Robust predictive tracking control for mobile robots with intermittent measurement and quantization," IEEE Trans. Ind. Electron., vol. 68, no. 1, pp. 509-518, Jan. 2021.

[3] J. Hua, C. Li, and H.OL. Shen, "Distributed learning of predictive structures from multiple tasks over networks," IEEE Trans. Ind. Electron., vol. 64, no. 5, pp. 4246-4256, May 2017

[4] Z. Li, C. Zhang, P. Zeng, H. Yu, S. Li, and J. Bian, "Control of a gridforming inverter based on sliding-mode and mixed $\mathrm{H}_{2} / \mathrm{H}_{\infty}$ control," IEEE Trans. Ind. Electron., vol. 64, no. 5, pp. 3862-3872, May 2017.

[5] M. Vazquez-Olguin, Y. S. Shmaliy, Choon Ki Ahn, and O. IbarraManzano, "Blind robust estimation with missing data for smart sensors using UFIR filtering," IEEE Sensors J., vol. 17, no. 6, pp. 1819-1827, Mar. 2017.

[6] K. Uribe-Murcia, Y. S. Shmaliy, C. K. Ahn, and S. Zhao, "Unbiased FIR filtering for time-stamped discretely delayed and missing data," IEEE Trans. Autom. Control, vol. 65, no. 5, pp. 2155-2162, May 2020.

[7] T. Liu, P. Garcia, Y. Chen, X. Ren, P. Albertos, and R. Sanz, "New predictor and 2DOF control scheme for industrial processes with long time delay," IEEE Trans. Ind. Electron., vol. 65, no. 5, pp. 4247-4256, May 2018

[8] C. Zhang, G. Wu, F. Rong, J. Feng, L. Jia, J. He, and S. Huang, "Robust fault-tolerant predictive current control for permanent magnet synchronous motors considering demagnetization fault," IEEE Trans. Ind. Electron., vol. 65, no. 7, pp. 5324-5334, Jul. 2018.

[9] J. Makhoul, "Linear prediction: A tutorial review," Proc. IEEE, vol. 63, no. 4, pp. 561-580, Apr. 1975.

[10] Y. S. Shmaliy, "An unbiased $p$-step predictive FIR filter for a class of noise free discrete-time models with independently observed states," Signal, Image Video Process., vol. 3, no. 2, pp. 127-135, Jun. 2009.

[11] F. Auger, M. Hilairet, J. M. Guerrero, E. Monmasson, T. OrlowskaKowalska, and S. Katsura, "Industrial applications of the Kalman filter: A review," IEEE Trans. Ind. Electron., vol. 60, no. 12, pp. 5458-5471, Dec. 2013.

[12] G. F. Basso, T. G. Silva De Amorim, A. V. Brito, and T. P. Nascimento, "Kalman filter with dynamical setting of optimal process noise covariance," IEEE Access., vol. 5, pp. 8385-8393, 2017.

[13] Y. S. Shmaliy, "Linear optimal FIR estimation of discrete time-invariant state-space models," IEEE Trans. Signal Process., vol. 58, no. 6, pp. 3086-3096, 2010.

[14] Y. S. Shmaliy and O. Ibarra-Manzano, "Time-variant linear optimal finite impulse response estimator for discrete state-space models," Int. J. Adapt. Contr. Signal Process., vol. 26, no. 2, pp. 95-104, 2012

[15] Y. S. Shmaliy, "An unbiased FIR filter for TIE model of a local clock in applications to GPS-based timekeeping," IEEE Trans. on Ultrason., Ferroel., Freq. Contr., vol. 53, no. 5, pp. 862-870, May 2006.

[16] W. H. Kwon and S. Han, Receding Horizon Control: Model Predictive Control for State Models. London, UK: Springer, 2005.

[17] O. K. Kwon, W. H. Kwon, and K. S. Lee, "FIR filters and recursive forms for discrete-time state-space models," Automatica, vol. 25, no. 5, pp. 715-728, 1989.

[18] Y. S. Lee, S. H. Han, and W. H. Kwon, " $H_{2} / H_{\infty}$ FIR filters for discrete-time state space models," Int. J. of Contr. Autom. and Systems., vol. 4, no. 5, pp. 645-652, Oct. 2006.

[19] C. K. Ahn, Y. S. Shmaliy, and S. Zhao, "A new unbiased FIR filter with improved robustness based on Frobenius norm with exponential weight," IEEE Trans. Circuits Syst. II Express Briefs., vol. 65, no. 4 , pp. 521-525, Apr. 2018.

[20] C. K. Ahn, S. Zhao, Y. S. Shmaliy, and H. Li, "On the $\ell_{2}-\ell_{\infty}$ and $H_{\infty}$ performance of the continuous-time deadbeat $\mathrm{H}_{2}$ FIR filter," IEEE Trans. Circuits Syst. II Express Briefs., vol. 65, no. 11, pp. 1798-1802, Nov. 2018. 
[21] S. Zhao, Y. S. Shmaliy, and F. Liu, "Optimal FIR filter for discretetime LTV systems and fast iterative algorithm," IEEE Trans. Circuits Syst. II, Express Briefs (to be published).

[22] W. M. Haddad, D. S. Bernstein, and D. Mustafa, "Mixed-norn $H_{2} / H_{\infty}$ regulation and estimation: The discrete-time case," Syst. \& Contr. Lett., vol. 16 , pp. 235-247, 1991.

[23] M. D. S. Aliyu and E. K. Boukas, "Discrete-time mixed $H_{2} / H_{\infty}$ nonlinear filtering," Int. J. Robust Nonlinear Cont., vol. 21, pp. 12571282, 2011.

[24] Z. Tan, Y. C. Soh, and L. Xie, "Envelope-constrained $H_{2}$ FIR filter design," Circ. Syst. Signal Process., vol. 18, no. 6, pp. 539-551, 1999.

[25] B.-S. Chen and J.-C. Hung, "Fixed-order $H_{2}$ and $H_{\infty}$ optimal deconvolution filter designs," Signal Process., vol. 80, no. 2, pp. 311-331, 2000.

[26] S. Wang, L. Xie, and C. Zhang, " $H_{2}$ optimal inverse of periodic FIR digital filters," IEEE Trans. Signal Process., vol. 48, no. 9, no. 9, pp. 2696-2700, 2000.

[27] S. Wang, L. Xie, and C. Zhang "Mixed $H_{2} / H_{\infty}$ deconvolution of uncertain periodic FIR channels," Signal Process., vol. 81, no. 10, pp. 2089-2103, Oct. 2001.

[28] K. Zhou, J. C. Doyle, and K. Glover Robust and Optimal Control, Upper Saddle River, NJ: Prentice-Hall, 1996.

[29] B. Hassibi, A. H. Sayed, and T. Kailath, Indefinite-Quadratic Estimation and Control: A Unified Approach to $H^{2}$ and $H^{\infty}$ Theories, Philadelphia, PA: SIAM, 1999.

[30] F. L. Lewis, L. Xie, and D. Popa, Optimal and Robust Estimation with an Introduction to Stochastic Control Theory, 2nd Ed., Boca Raton, FL: CRC Press, 2008.

[31] C. Scherer and S. Weiland, Linear Matrix Inequalities in Control, Stuttgart, DE: University of Stuttgart, 2015.

[32] Y. S. Shmaliy, "An iterative Kalman-like algorithm ignoring noise and initial conditions," IEEE Trans. Signal Process., vol. 59, no. 6, pp. 2465-2473, Jun. 2011.

[33] Y. S. Shmaliy, S. Zhao, and C. K. Ahn, "Unbiased FIR filtering: an iterative alternative to Kalman filtering ignoring noise and initial conditions," IEEE Contr. Syst. Mag., vol. 37, no. 5, pp. 70-89, 2017.

[34] J. Ortega-Contreras, Eli Pale-Ramon, Y. S. Shmaliy, and Y. Xu, A novel approach to H2 FIR prediction under disturbances and measurement errors, IEEE Signal Process. Lett., vol. 28, pp. 150-154, 2021.

[35] T. Kailath, A. H. Sayed, and B. Hassibi, Linear Estimation, Upper Saddle River, NJ: Prentice Hall, 2000.

[36] A. E. Bryson and L. J. Henrikson, "Estimation using sampled data containing sequentially correlated noise," J. Spacecraft Rockets, vol. 5, no. 6, pp. 662-665, 1968.

[37] M. G. Petovello, K. O'Keefe, G. Lachapelle, M. E. Cannon, "Consideration of time-correlated errors in a Kalman filter applicable to GNSS," J. of Geodesy, vol. 83, no. 1, pp. 51-53, 2009.

[38] Y. Xu, Y. S. Shmaliy, C. K. Ahn, G. Tian, and X. Chen, "Robust and accurate UWB-based indoor robot localization using integrated EKF/EFIR filtering, IET Radars, Sonars \& Navigation, vol. 12, no. 7, pp. 750-756, 2018.

\section{Creative Commons Attribution License 4.0 (Attribution 4.0 International, CC BY 4.0)}

This article is published under the terms of the Creative Commons Attribution License 4.0 https://creativecommons.org/licenses/by/4.0/deed.en US 